38. Lebedeva E. V., Gorokhov A. S., Schastnyy E. D., Repin A. N., Simutkin G. G. [et al.] Time course of cognitive dysfunction and biochemical marker of CNS lesions $\mathrm{S} 100 \mathrm{~B}$ in coronary artery bypass graft. Byulleten' sibirskoj mediciny. 2018:17(2):72-84.

https://doi.org/10.20538/1682-0363-2018-4-72-84

39. Golubev A. M., Kuzovlev A. N., Antonova V. V., Zakharchenko V. E., Petrova M. V. [et al.] Molecular biomarkers for prediction of neurological outcome after sudden circulatory arrest (review). General reanimatology. 2018;14(3):6881. https://doi.org/10.15360/1813-9779-2018-3-68-81

40. Avrushchenko M. S., Ostrova I. V. Postresuscitative changes of brainderived neurotrophic factor (BDNF) protein expression: association with neuronal death. General reanimatology. 2017;13(4):6-21.

https://doi.org/10.1016/j.resuscitation.2017.08.196
41. Ivkin A. A., Grigoriev E. V., Shukevich D. L. Diagnostics of cognitive dysfunction in patients in the intensive care wards. Vestnik anesteziologii i reanimatologii. 2018;15(3):47-55. https://doi.org/10.21292/2078-5658-2018-15-3-47-55

42. Chen H., Wu Y., Huang G., He W., He W. [et al.] Low tri-iodothyronine syndrome is associated with cognitive impairment in patients with acute ischemic stroke: a prospective cohort study. Am. J. Geriatr. Psychiatry. 2018;26(12):12221230. https://doi.org/10.1016/j.jagp.2018.07.007

43. Huang S., Zhao J., Huang D., Zhuo L., Liao S. [et al.] Serum miR-132 is a risk marker of post-stroke cognitive impairment. Neurosci. Lett. 2016;615:102-106. https://doi.org/10.1016/j.neulet.2016.01.028

\begin{abstract}
About authors: tel.: +79111395559; e-mail: atmspb@mail.ru

tel.: +79119111055; e-mail: alexey-iv@yandex.ru

tel.: +79818278785; e-mail: aet-spb@yandex.ru

tel.: +79043361100; e-mail: aomakarov@mail.ru

(C) Akter M., 2019

UDC 616.381-072.1.995.121

DOI - https://doi.org/10.14300/mnnc.2019.14142

ISSN - 2073-8137
\end{abstract}

Efimova Maria Yurevna, CMedSc, Assistant of Professor of the Department of neurology and psychiatry; neurologist of the Department of rehabilitation; tel.: +79052705565; e-mail: medice_levsha@mail.ru

Ivanova Natalia Evgenevna, MD, Professor, Chief researcher; tel.: +79112187149; e-mail: ivamel@yandex.ru

Alekseeva Tatyana Mihaylovna, MD, Associate Professor, Head of the Department of neurology and psychiatry;

Ivanov Aleksey Yurevich, MD, Professor of the Department of cardiovascular surgery;

Tereshin Alexey Evgenevich, CMedSc, Head of the Rehabilitation Department;

Makarov Artem Olegovich, CMedSc, Head of the Neurological Department № 2;

Reshetnik Dmitrii Aleksandrovich, MD, Chief physician; tel.: +78124097500; e-mail: rda1212@yandex.ru

\title{
EXTRACTION OF TEXTURE FEATURES FROM MEDICAL IMAGES: OSTEOARTHRITIS CASES REVIEW
}

Akter $M$.

University of Bedfordshire, Luton, United Kingdom

\section{ВЫАЕАЕНИЕ ТЕКСТУРНЫХ ОСОБЕННОСТЕЙ ААННЫХ ИЗ РЕНТГЕНОВСКИХ ИЗОБРАЖЕНИЙ ПРИ ОСТЕОАРТРОЗЕ}

\author{
М. Актер
}

\section{Университет БеАфорАшира, Аутон, Великобритания}

Texture features of osteoarthritis quantitatively represent patterns of interest in image analysis and interpretation in medicine. Texture features can widely vary so that the analysis can lead to interpretation errors and undesirable consequences. In such cases, finding of informative features becomes problematic. In medical imaging, the texture features of bones were useful for representing variations in patterns of pixel intensity, which were correlated with pathological changes. In this paper, we review existing approaches to extracting the texture features and conclude on usability, including machine learning.

Keywords: texture features, osteoarthritis, medical imaging, pattern recognition, machine learning

Точная визуализация и интерпретация рентгенологической картины текстуры остеоартроза представляет интерес в современных условиях. Особенности изменений текстуры костной ткани могут сильно варьировать, и поэтому проводимый компьютерный анализ может приводить к ошибкам в интерпретации данных и к неправильной постановке диагноза. В таких случаях поиск информативных признаков становится проблематичным. В медицинской визуализации особенности текстуры строения кости чрезвычайно важны для представления вариаций паттернов 
интенсивности пикселей, которые коррелируют с патологическими изменениями в костной ткани. Рассмотрены существующие подходы к получению данных и распознаванию патологических процессов с привлечением искусственного интеллекта в медицине.

Ключевые слова: текстурные особенности, остеоартроз, медицинская визуализация, распознавание образов, машинное обучение

For citation: Akter M. EXTRACTION OF TEXTURE FEATURES FROM MEDICAL IMAGES: OSTEOARTHRITIS CASES REVIEW. Medical News of North Caucasus. 2019;14(3):571-576. DOI - https://doi.org/10.14300/mnnc.2019.14142

Для цитирования: Актер М. ВЫДЕЛЕНИЕ ТЕКСТУРНЫХ ОСОБЕННОСТЕЙ ДАННЫХ ИЗ РЕНТГЕНОВСКИХ ИЗОБРАЖЕНИЙ ПРИ ОСТЕОАРТРОЗЕ. Медицинский вестник Северного Кавказа. 2019;14(3):571-576. DOI - https://doi.org/10.14300/mnnc.2019.14142

GMDH - Group Method of Data Handling

OA - Osteoarthritis
RFCLM - Random Forest Regression Voting Constrained Local Model
F eature extraction is the most important function of different applications in image processing. The texture is the main characteristic used to identify the objects and areas of interest in an image, where the image likes to be a satellite image, photomicrograph, or aerial photograph. A feature is a characteristic that represents a certain visual property of image locally or globally for the objects, regions, or the whole images. Elements also represent the structural properties of objects visible at the micro and macro scales. Spatial distributions of variations in the properties are represented by textures [1, 2]. There are different applications where texture features are used in several ways for extracting the features of image, such as image processing and content-based image retrieval. Texture features are designed to quantitatively describe image patterns of interest for pattern analysis, interpretation, and recognition. Unexplained variations of designed features very often lead to interpretation errors and undesirable consequences. Nevertheless, texture features have been created capable of describing patterns of pixel intensity variations, which were highly correlated with structural alterations of biological tissues [3].

In X-ray medical imaging, bone pathology, such as Osteoarthritis (OA), is characterized by the loss of joint cartilage and development of bone spurs, which causes pain and impaired movement [4]. Cartilage is the connective and protective tissues that are part of end bone in a joint. Healthy cartilage prevents the bones from rubbing each other and allows easy bone glide, but erupted cartilage cannot protect the bone from rubbing them [4]. Primary and Secondary are two types of OA, Primary OA seen in older adults due to obesity, athletics with rheumatoid arthritis, diabetes and injury, etc. whereas secondary OA tends to show up in aged people because of aging and reason for genetic.

OA is typically diagnosed when patients have developed symptoms such as joint pain, and the pathological changes are visually recognizable in images [4]. At an early stage, the changes in bone structure can be evaluated with magnetic resonance imaging, which, however, is costly and time-consuming, and so cannot be widely used. Alternative X-ray technologies provide cost-efficient solutions. Sometimes there are some distortions in X-ray images that make obstacles to detect early osteoarthritis. When the findings of $x$-ray images are not visible doctor recommends the patient for $\mathrm{MRI}[4,5,6]$. Many people may not afford this MRI because of its expenses. There- fore, we have tried to implement a Deep Learning paradigm method that helps in the diagnosis of the disease to some extent.

A method has been proposed for automated detection of OA in X-ray images which employed the ordinary Wavelet, Fourier, Haralick, and Chebyshev transform [6]. However, it has been found that some of the generated features were affected by the noise. The processing was done by three steps, such as feature extraction, processing of image and classification through backpropagation neural network. The author concluded with $66.6 \%$ of classification rate.

The analysis shows that additional efforts are required to decide on the structure of texture features, which could provide the best recognition accuracy. The majority of the existing techniques use a try-and-see strategy for finding such solutions. In this paper, we analyze strategy of designing the texture features with the desired properties, which are learned from a given set of images. The desired characteristics are discovered directly from the labeled images, which are prepared by experts that why additional efforts are not required. To achieve this aim we use a Machine Learning method based on Deep Learning paradigm known as Group Method of Data Handling, delivering efficient solutions under realistic conditions which are determined, in particular, by noise and natural variations in images.

$\mathrm{OA}$ is a joint disease that occurs from the breakdown of the underlying bone and the joint cartilage. Structural and mechanical alterations are the main features of the OA. Architectural features such as osteophytes, trabeculae, denudation, Bone Marrow Leions, orientation changes, Joint Space Narrowing, cysts, attrition of articular surfaces, and inflammation of the synovium are linked to the mechanical stress [7]. Alternatively, the mechanical alterations occur with the further progression of biomechanical diseases.

Osteophytes are bony spurs that grow on the bone, particularly in the articular cortical surface of the spine and around the margins of the joint. It has been discussed that the osteophytes are a pro-inflammatory reaction that damages the tendons and cartilage tissues of the bone [7]. Typically, the osteophytes occur during the development of osteoarthritis. Joint space narrowing occurs from the degradation of the cartilage tissue and overuse of the joint. It can also happen from other risk factors such as aging, obesity, and weakness of the muscle.

In osteoarthritis knee, the surface of the weight-bearing subchondral trabeculae will be thickening and change the direction in conformity with Wolff's Law, which improves the strain caused through focal pres- 
sure [7]. The trabeculae are sturdy to moving down the leg on a healthy aligned joint but it also weak to forces the tension. During the early OA, the horizontal trabeculae start to become thicken through the shift in pressure and weight to grip the extra powers of tension whereas the vertical trabeculae appear in the last stages to control the alleviate pressure from loss of joint space.

Typically, tibia spine fracture is relatively sporadic injuries that occur in the skeletally immature knee, and it also becomes irregular in shape, rough, and spiked. The main reason for the tibia spine fracture is the formation of the new bone across the knee. Recently, the disease organization is focused on plummeting functional and pain impairment, but there is a range of interventions that are non-pharmacological and pharmacological, including nutriceutical and orthotics can be used. It is also important to losing the overweight and taking regular exercise to control osteoarthritis. Currently, there is no treatment shown to slow down the progression of OA disease [7].

According to $[8,9]$, texture has been defined as that; it is a continual form of information or an organized structure that is committed at regular intervals. Moreover, texture illustrates characteristics of surface and a visible appearance of the object which means that it would demonstrate the physical magnitude of the elementary parts of the object by size, shape, amount, arrangement. Texture feature extraction is a process that goes through various types of analysis, and the fundamental tasks of that analysis are to collect features through the scrutinized process of extraction [10].

Indirectly statistical techniques describe the texture feature based on non-deterministic properties that deal with the connections between the gray level images. This statistical technique is utilized to examine the spatial dispersion of gray values through processing neighborhood features at each point in the image. It is also inferring a group of information from the dispersions of the neighborhood features. This method is classified by second-order pair of pixels. The higher and secondorder evaluates properties of at least two pixels esteems happening in particular areas for each other. The most common statistical features are resulting from the cooccurrence matrix for investigating the texture feature [8].

To extract the second-order information to complete an image, the GLCM is one of the most famous and stable statistical devices in this field. This device produced the information without any kinds of errors, i.e., it provides the numeric numbers on rows and columns equally where the grey levels and pixel of the images and its surfaces could be highlighted without any kinds of errors [22]. The frequency of the grey level has been highlighted by the GLCM method, which is a matrix, and the rate of that grey level has a connectedness between the grey levels where the activities of that grey level could be seen within the specific area of investigation [8]. The amount of electromagnetic information transmitted to produce an image, the GLCM set out a tabular form of various kinds of combinations of grey levels in different stages of the section of the image [11].

To set out the pixel of interest in terms of measuring the variation of intensity, the contents of GLCM must go through a calculative process, which is known as the texture feature calculation [12]. Two parameters could calculate the formative distance between the pixel pair and other relevant features that are closely related to this measure. There are many directions in which the measure could be done, but most commonly used guidelines are the 'four directions' [11]. Contrast and entropy are the most used features in GLCM where fourteen of them are available to measure the correlation of the features [9].
Nowadays, the most important statistical features are co-occurrence matrix and texture features, which were introduced by Haralick [13]. It has used two steps for extracting the texture feature. The co-occurrence matrix is registered in the initial stage, and then features of the texture are computed based on co-occurrence matrix in the final step. In the application of image analysis this technique is the most popular and widely used for remote sensing to biomedical technology.

For an image, thirteen texture features extracted by the Haralick texture from GLCM. The essential features of image classification are Contrast, Energy, Entropy, and Directional Moment [8]. Entropy and contrast are the most important statistical parameters among the thirteen originally texture features [13].

A set of complex polynomials is introduced by Zernike movement that forms a complete set of orthogonal over the interior circle unit. The moments of Zernike are the projection of image function against these orthogonal functions. Order $\mathrm{n}$ Zernike moment with repetition $\mathrm{m}$ and $f(x, y)$ continuous image function vanishes outside the unit circle is for computing the Zernike moments, the image center is taken as the origin, and the coordinates of the pixel are plotted to the range of circle unit such as $x^{2}+y^{2} \leq 1$. That outside falling pixel of the unit circle is not used in the computation [13].

In machine learning, the essential problem is a classification issue that happened for the presence of irregular statistics or features [14]. The classification of image alludes to the formation of feature classes utilizing semi-supervised, supervised and unsupervised technique. The substance of feature classes is to coordinate different inputs to a specific type of feature for recognition. According to [15], the supervised classification is based on known information, whereas unsupervised involved the data sample that is not known. The semi-supervised classification included both known and unknown data samples [16]. In extracting texture features from X-ray images, supervised classification methods are adopted because it works with the presence database with known images and the advantage of supervised learning is that the execution of the system is speedier because of prior learning knowledge from labeled datasets.

A random forest is a collection of tree-structured classifiers. According to [17], the accuracy of regression and classification can be gained by utilizing ensembles of trees where each tree in the outfit is developed as per an arbitrary parameter. The last expectations can be achieved by combining over the group. As the tree-structured predictors are the base constituents of the group and every one of these trees is built using an injection of arbitrariness. These techniques are called random forest [18], which have been successfully used in the medical domain [19-23].

It is capable of inducing polynomial neural networks from noisy data, which is based on the assumption that the noise is Gaussian. To solve a complex problem, including classification problems through self-organization [8], Ivakhnenko developed the polynomial neural networkGMDH initially. Ivakhnenko has suggested a Group Method of Data Handling (GMDH) that is capable of inducing neural networks from noise data Gaussian distributed. Based on an evolutionary strategy, the GMDH generates populations or layers of neurons. During learning the GMDH grows more new population of neurons, and the number of layers or complexity of the network increases while a predefined criterion is met [23]. After that, it trains and selects those neurons which provide the best classification. The fully connected neural-network classifiers user less observable than a set of short-term polynomials [24- 
26]. In GMDH system, a particular criterion is computed based on validation of the data set that helps to evaluate the performance of the neurons. Subsequently, GMDH system sums up well because of the appropriation of noise in information, which is accepted as Gaussian. Besides, GMDH is a set of short-term polynomials that are not difficult to observe by specialists [27].

The quantitative method is mostly essential for measurements of extracting the OA features on radiographs. In [28], it has been used the JSN and attrition for the latter grading score that influences through the growth of tibia plateaus during the OA. The reliability of this method was found to be slow due to relatively simple measurement with few confounding factors. In [29], a method has been focused on specific features of the disease for spine and hip that occur with inter-observer reliability ranging from $0.8-$ 0.93 for minimal JSW and 0.66 for osteophytes. According to the severity and feature of the diseases, the quantitative method has been divided into two stages, such as quantitative measurement and semi-quantitative measurement. In [30], a method has been used to avoid erroneous measures and miss-classification of the OA disease. It is also useful for accurately measuring the progression of $\mathrm{OA}$ disease. However, due to the old technology, these methods have been noted to be cumbersome and inefficient for extracting and measuring the features from X-ray images [31].

A method for automated detection of $\mathrm{OA}$ in X-ray images has been described in [32]. The proposed method has employed common Wavelet, Fourier, Haralick, and Chebyshev transform. In their work, a large set of image features are extracted first, and then the most informative features are selected. Radiologists, however, were limited to interpret features that were extracted from the transforms, and for this reason, the required markers were selected empirically by testing all combinations of descriptors [19]. The contribution of each generated feature to the detection accuracy was estimated, and then the resultant vector of 1470 components has been designed by using a set of 210 descriptors for seven different image transforms. However, it has been found that some of the generated features were affected by noise. The most informative features were finally selected by using a Fisher score calculated for each image descriptor [32].

Rotation-invariant features have been proposed based on Zernike orthogonal moments of the image [32, 34]. A set of rotational invariant features that are magnitudes of the moment of the orthogonal complex in an image known as Zernike moments. Scale invariance was obtained by using regular geometrical moments of the image concerning these parameters. An image reconstruction method using highest-order Zernike moments has been developed for solving classification problems. The evaluation of the original image scrutinized the reconstructed image quality. The orthogonal property of the Zernike moments has made the proposed feature selection method feasible. Texture features were weighted according to their contribution to the classification. It is important to note that the Zernike moments, being computationally efficient, have a rotation invariance property, and thus can be used as rotation-invariant features for image representation.

In work [35], it has been used Gober Filter methods for automated segmentation of impaired joint space on osteoarthritis x-ray images. In their work the whole method is divided into two steps, the first step is to segment right and left knee, and this step is divided into four experiments: the first experiment uses the center of mass, second uses only templates matching, third uses CLAHE \& template matching and fourth combines all the above experiments. The second step is to find JSA which is divided into two experiments, first uses row sum graph \& center of mass method \& second uses Gabor filter, row sum graph \& center of mass method. The first four steps of results gave sufficiently good results with $78 \%$ accuracy, and in the second steps of experiment 2 gave $80.63 \%$ accuracy for the right knee and $78.37 \%$ for left knee.

A computer-aided automated system has been proposed to recognize the bone outline of the knee joint for osteoarthritis disorder [7]. The Random Forest Regression Voting Constrained Local Model (RFCLM) and statistical shape model have been used for analyzing the automated system. Two different classifiers were used to identify the healthy \& affected OA and to distinguish among the texture in knee bone. The computed features were pixel features, shape features and texture features. The experiment is concluded with sufficient good outcomes and $78.9 \%$ accuracy.

In work [36], it has been proposed a joint detection algorithm to examine the X-ray image and used feature extraction techniques such as the first four moments, Zernike features, and multi-scale histograms. The rules of the weighted nearest neighbor have utilized to classify the extracted features and the detection algorithm used to find out the joint and isolate this joint from the rest of the images. In conclusion, $80 \%$ of moderate osteoarthritis was separated from ordinary $\mathrm{OA}$, and $70 \%$ of the lowest OA was separated from typical.

In paper [37], it has been proposed an edge detection algorithm and feature extraction techniques such as patella cartilage, femur, and tibia to examine the edges from $\mathrm{X}$-ray images in OA. From this proposed algorithm, the author achieved adequately excellent outcomes and is exceptionally successful in noisy and blur images.

In work [38], it has been proposed an algorithm for various image segmentation to identify the problems and abnormalities related to bone structures. The discrete level gives very quick and great outcomes among these three segmentations. In [39], it has been proposed various techniques for image processing to detect the width of joint space based on $x$-ray images of bone. This image processing technique is histogram equalization, thresholding, contrast enhancement, and canny edge detection algorithm. In this paper, the width of joint space is contrasted with normal width in the bone $x$-ray image. This image is called to be an osteoarthritis case or standard case.

In paper [40], a backpropagation technique has been used for analyzing the sternness of OA ailment. The processing was done by three steps, such as feature extraction, processing of image and classification through backpropagation neural network process. The author concluded with $66.6 \%$ of classification rate.

In work [41], it has been proposed computer-aided diagnosis techniques to detect osteoarthritis in X-ray images. The Haralick feature extraction method and SVM classifier have been used for computation and detection of OA. This paper is concluded with sufficient good outcomes and $78 \%$ accuracy in the analysis of bone disorders caused by osteoarthritis.

The computer-assisted method [38] has been proposed for automatically detecting the OA from bone $\mathrm{x}$-ray images. The better quality image is obtained from which mean \& variance is calculated using the neighboring pixel method. In their work image is enhanced using a Gaussian low pass filter and stretched using contrast stretch technique. The Euclidean distance formula is used in the binary format of the image to calculate the JSW, which is compared with the normal value that concludes among 80 images; the success rate was up to $90 \%$, and $10 \%$ images failed as thresholding was not clear. 
Image analysis techniques for identifying the connections between the clinical gauge and radiographic findings [6]. In this method, the WND-CHRM algorithm used Shape, Haralick, Statistical, and Tamura feature extraction techniques and weighted Nearest Neighbor used as a classifier to compute the features. The experiment showed a satisfactory correlation between clinical gauge and radiographic findings.

In paper has been used a Local Ternary Pattern algorithm for computing the features from digital X-ray images and Support Vector Machine Classifiers have used to classify them. To evaluate this algorithm's performance using $50 \mathrm{x}$-ray samples. Finally, the experiment provides $91.66 \%$ specificity \& $80 \%$ sensitivity from Polynomial and Linear function, and $94.59 \%$ specificity \& $66.66 \%$ sensitivity from RBF

In work [42], it has been developed a k-mean technique for automatic clustering of $r$-ray images and applied hierarchical and large feature vector methods on $\mathrm{k}$-means clustering. The bunching was completed on multilevel features of $\mathrm{x}$-ray images. The experiment showed that the blend of hierarchical clustering and k-means clustering provides a satisfactory accuracy level. From this work, it has been seen that a decent number of researchers worked on x-ray bone images for characterization and detection of osteoarthritis through various methodologies and predicted some promising outcomes by their data samples. But still there have a chance to build up a robust algorithm for providing the maximum recognition accuracy. The majority of the existing techniques use a try-and-see strategy for finding such a decision. Thus, we propose a new method for designing the texture features with the desired properties, which are learned directly from the labeled images, which are prepared by experts, and so additional efforts are not required.

Conclusions. The bone pathologies can be detected by using Machine Learning methods delivering efficient solutions under realistic conditions that are determined by noise and natural variations in images. The most suitable methods known from the related literature are associated with designing new features having the desired properties for representing images that can be implemented within the Deep Learning paradigm. Group Method of Data Handling, which has pioneered Deep Learning was efficient for solving recognition problems. Texture features can efficiently represent patterns of interest for purposes of image analysis and interpretation. However, texture features can vary so mainly that the investigation leads to interpretation errors and undesirable consequences. In such cases, finding informative features become problematic. Nevertheless, we conclude that the texture features were useful for representing variations in patterns of pixel intensity, which were correlated with pathological changes.

Disclosures:

The author declares no conflict of interest.

\section{References}

1. Depeursinge A., Al-Kadi O., Mitchell J. Biomedical Texture Analysis: Fundamentals, Tools and Challenges. Elsevier Science \& Technology Books. 2017.

2. Haralick R. M., Shanmugam K., Dinstein I. Textural features for image classification. IEEE Transactions on Systems, Man, and Cybernetics SMC. 1973;3(6):610-621.

3. Ghosh A., Sarkar A., Ashour A. S., Balas-Timar D., Dey N. Grid color moment features in glaucoma classification. International Journal of Advanced Computer Science and Applications. 2015;6(9):99-107.

4. Shamir L., Ling S. M., Scott W. W. Jr. Knee x-ray image analysis method for automated detection of osteoarthritis. IEEE Trans. Biomed. Eng. 2009;56(2):407-415.

5. Shamir L. A Computer analysis method for correlating knee X-ray with continuous indicators. International Journal of Computer Assisted Radiology \& Surgery (CARS). 2011:6:699-704.

6. Shamir L., Ling S. M., Jr. W. W. S., Bos A., Orlov N. [et al.] Knee x-ray image analysis method for automated detection of osteoarthritis. IEEE Transactions on Biomedical Engineering. 2009;56(2):407-415.

7. Thomson J. Algorithm for automatic analysis of radiographs of the knee with application in diagnosis and monitoring of osteoarthritis. Doctor of Philosophy. University of Manchester. 2016.

8. He D., Wang L., Guibert J. Texture feature extraction. Pattern Recognition Letters. 2003;6(4):269-273.

9. Li Z., Shi K., Dey N., Ashour A. S., Wang D. Rule-based backpropagation neural networks for various precision rough set presented kansei knowledge prediction: a case study on shoe product form features extraction. Neural Computing and Applications. 2017;28(3):613-630.

10. Xiao J., He C. Dynamic Classifier Ensemble Selection Based on GMDH, Computational Sciences and Optimization. International Joint Conference. 2009:731.

11. Li Z., Shi K., Dey N., Ashour A. S., Wang D. Rule-based backpropagation neural networks for various precision rough set presented kansei knowledge prediction: a case study on shoe product form features extraction. Neural Computing and Applications. 2017;28(3):613-630.
12. Wang D., He T., Li Z., Cao L., Dey N. Image feature-based effective retrieval employing improved parameter and structure identification of adaptive neuro-fuzzy inference system. Neural Computing and Applications. 2016;29(4):10871102.

13. Haralick R. M., Shanmugam K., Dinstein I. Textural features for image classification. IEEE Transactions on Systems, Man, and Cybernetics SMC. 1973;3(6):610-621.

14. Paiva A. R. C., Tasdizen T. Detection of Salient Image Points Using Principal Subspace Manifold Structure. Pattern Recognition (ICPR). 20th International Conference. 2010:1389.

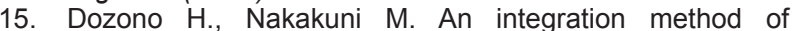
multi-modal texture method using supervised pareto learning. Neural Networks. IJCNN. IEEE International Joint Conference. 2008:602.

16. Yu J., Wang M., Tao D. Semisupervised Multiview Distance Metric Learning for Cartoon Synthesis. Image Processing IEEE Transactions. 2012;21(11):4636-4648.

17. Breiman L. Random forests. Machine Learning. 2001; 45(1):5-32.

18. Boniatis I., Costaridou L. Cavouras D., Kalatzis I., Panagiotopoulos. Osteoarthritis severity of the hip by computeraided grading of radiographic images. Medical and Biological Engineering and Computing. 2006;44(9):793803.

19. Schetinin V., Jakaite L., Krzanowski W. Prediction of survival probabilities with Bayesian Decision Trees. Expert Systems with Applications. 2013;40(14):5466-5476.

20. Jakaite L., Schetinin V., Maple C., Schult J. Bayesian decision trees for EEG assessment of newborn brain maturity. The 10th Annual Workshop on Computational Intelligence UKCI. 2010. https://doi.org/10.1109/UKCl.2010.5625584

21. Jakaite L., Schetinin V., Schult J. Feature extraction from electroencephalograms for Bayesian assessment of newborn brain maturity. In: 24th International Symposium on Computer-Based Medical Systems (CBMS). 2011:1-6. https://doi.org/10.1109/CBMS.2011.5999109

22. Jakaite L. Schetinin V., Maple C. Bayesian assessment of newborn brain maturity from two-channel sleep electroencephalograms. Computational and Mathematical Methods in Medicine. 2012:1-7. https://doi.org/10.1155/2012/629654 
23. Schetinin V., Jakaite L., Jakaitis J., Krzanowski W. Bayesian decision trees for predicting survival of patients: A study on the US national trauma data bank. Computer Methods and Programs in Biomedicine. 2013;111(3):602612. https://doi.org/10.1016/j.cmpb.2013.05.015

24. Madala H., Ivakhnenko A. G. Inductive Learning Algorithms for Complex Systems Modeling. CRC Press Inc.: Boca Raton. 1994.

25. Muller J. A., Lemke F. Self-Organizing Data Mining. Extracting Knowledge from Data. Trafford Publishing, Canada. 2003.

26. Miller J. A., Lemke F., Ivakhnenko A. G. GMDH algorithms for complex systems modeling. Math and Computer Modeling of Dynamical Systems. 1998;4:275-315.

27. Schetinin V., Schult J. A neural-network technique to learn concepts from electroencephalograms. Theory in Biosciences. 2005;124(1):41-45. https://doi.org/10.1016/j.thbio.2005.05.004

28. Qazi A. A., Folkesson J., Pettersen P. C., Karsdal M. A., Christiansen C., Dam E. B. Separation of healthy and early osteoarthritis by automatic quantification of cartilage homogeneity. Osteoarthritis and Cartilage. 2007;15(10):11991206.

29. Hanna F. S., Teichtahl A. J., Wluka A. E., Wang Y., Urquhart D. M. [et al.] Women have increased rates of cartilage loss and progression of cartilage defects at the knee than men: a gender study of adults without clinical knee osteoarthritis. Menopause. 2009;19:666-670.

30. Manjunath B. S., Ma W.-Y. Texture Features for Image Retrieval, IBM T.J. Watson Research Center, and Image Database: Search and Retrieval of digital Imagery. 2002:313344.

31. Umbaugh S. E. Computer Imaging: Digital Image Analysis and Processing, Taylor and Francis Group, 2005.

32. Bouridane A., Tahir M. A., Kurugollu F. Accelerating the Computation of GLCM and Haralick Texture Features on recon gurable Hardware. Int. Conf. on Image Processing. 2004;5:2857-2860.
33. Khotanzad A., Hong Y. H. Invariant image recognition by zernike moments. IEEE Trans. Pattern Anal. Mach. Intell. 1990;12(5):489-497.

34. Teague M. R. Image analysis via the general theory of moments. Journal of the Optical Society of America. 1980;70(8):920-930

35. Anifah L., Purnama K., Hariadi M., Purnomo M. Automatic Segmentation of Impaired Joint Space Area for Osteoarthritis Knee X-ray Image using Gabor Filter based Morphology Process. The Journal of Technology \& Science. 2011;22(3):159-165

36. Lior S., Ling S., Scott W., Orlov N., Macura T. [et al.] Knee $X$-ray Image Analysis Method of Osteoarthritis. IEEE Transactions on Biomedical Engineering. 2008;16(6):306310.

37. Bandyopadhyay S. An Edge Detection Algorithm for Human Knee Osteoarthritis Images. Journal of Global Research in Computer Science. 2011;2(4):484-487.

38. Sharma P., Singh J. A Novel Approach towards X-ray Bone Image Segmentation using Discrete Step Algorithm. International Journal of Emerging Trends \& Technology in Computer Science. 2013;2(5):191-195.

39. Kubakaddi S., Urs N. Detection of Knee Osteoarthritis by Measuring the Joint Space Width in Knee X-ray Images. International Journal of Electronics \& Communication. 2015;3(4):18-21.

40. Pratiwi D., Santika D., Pardamean B. An Application of Back propagation Artificial Neural Network Method for Measuring the Severity of Osteoarthritis. International Journal of Engineering \& Technology. 2011;11(3):110-115.

41. Subromoniam M., Barani S., Rajini V. A non-invasive computer aided diagnosis of osteoarthritis from digital X-ray images. Biomedical Research. 2015;26(4):721-729.

42. Ray C., Sasma K. A New Approach for Clustering of X-ray Images. International Journal of Computer Science Issues. 2010;7(4):334-355

\section{About author}

Akter Mukti, MSc Computer Science, PhD student, School of Computer Science and Technology;

tel.: +41582743120; e-mail: mukti.akter@study.beds.ac.uk 